\title{
Invariants to Symmetrical Convolution with Application to Dihedral Kernel Symmetry
}

\author{
Jiří Boldyš and Jan Flusser \\ Institute of Information Theory and Automation of the ASCR \\ Pod vodárenskou věží 4, 18208 Praha 8, Czech Republic \\ \{boldys,flusser\}@utia.cas.cz \\ http://www.utia.cas.cz/
}

\begin{abstract}
We derive invariants to convolution with a symmetrical kernel in an arbitrary dimension. They are expressed in the Fourier domain as a ratio of the Fourier transform and of the symmetrical projection of the Fourier transform. In 2D and for dihedral symmetries particularly, we newly express the invariants as moment forms suitable for practical calculations. We clearly demonstrate on real photographs, that all the derived invariants are irreplaceable in pattern recognition. We further demonstrate their invariance and discriminability. We expect there is potential to use these invariants also in other fields, including microscopy.
\end{abstract}

Keywords: moment invariants, dihedral symmetry, symmetrical blur.

\section{Introduction}

In numerous practical situations we face image degradations which can be described as a convolution with a kernel $h$ which exhibits some kind of symmetry

$$
g=f * h,
$$

where $f$ is the original image and $g$ is the degraded image. A few examples can be out-of-focus blur, linear motion blur and atmospheric turbulence blur.

When dealing with out-of-focus blur, the blurring PSF is given by the shape of the aperture. If the aperture is fully open, the PSF is a cylinder-like function but if the aperture is open only partially, the PSF has a polygonal shape determined by number of aperture blades of the camera objective. Such PSF's have always $N$-fold rotation symmetry and sometimes also axial symmetry, see Fig. 1, This is why the blur invariants w.r.t. such PSF's are so important. A composition of $N$-fold rotation and axial symmetries is called dihedral symmetry. From the pattern recognition point of view, more constrained (i.e. "more symmetrical") kernels have less degrees of freedom and it can be expected that we can find more invariants to such a convolution and thus gain more discrimination power.

Invariants to $N$-fold symmetric blurs for $N=2$ were introduced in [1]. They have found numerous practical applications [2 -7]. Invariants to kernels with radial symmetry $(N=\infty)$ were derived in [8] and invariants for arbitrary $N$ were 


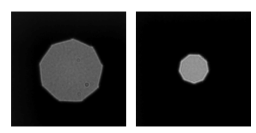

Fig. 1. Examples of convolution kernels caused by out-of-focus blur. Dihedral symmetry is very common due to shaping of the camera objective apperture by certain number of blades.

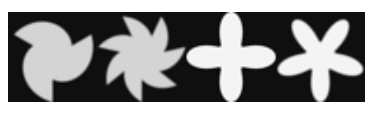

Fig. 2. Examples of various symmetries of $2 \mathrm{D}$ functions. From left to right having symmetries $C_{3}, C_{7}, D_{4}, D_{5}$.

proposed in [9] and most recently in [10]. They were derived in the spatial domain as functions of image moments. The invariants w.r.t. dihedral PSF's have not been published yet. This is the main contribution and novelty of the paper.

In the next sections we present a simple derivation of the general invariance to symmetrical convolution. Further on, we are more specific and we newly derive and test moment invariants to 2D dihedral symmetries. Moment invariants to 2D cyclic symmetries as a special case of the general theory are re-derived here (in a different way than in [10]) and put into the new context for illustration. In this paper we intensively rely on expansions of $2 \mathrm{D}$ functions into circular harmonics.

\section{Derivation of Invariants to Convolution Having Cyclic or Dihedral Symmetries}

\subsection{Preliminaries}

Two useful rotational image symmetries are cyclic and dihedral symmetries. If geometric transformations form a cyclic $C_{n}$, resp. dihedral $D_{n}$ group, then images having the cyclic (eq. to $N$-fold), resp. dihedral, symmetries remain under these transformations the same [11]. We will denote the symmetries also $C_{n}$, resp. $D_{n}$. Function $f(r, \varphi)$, where $r$ and $\varphi$ are polar coordinates, has the $C_{N}$ symmetry $\left(C_{n}\right.$ for particular $\left.n=N\right)$ if

$$
f\left(r, \varphi-\frac{2 \pi n}{N}\right)=f(r, \varphi) \quad \text { for } \quad n=0,1, \ldots, N-1 .
$$

$n$ more symmetry group elements are added in case of the dihedral group $D_{n}$ using reflection around an axis. Examples of images with both symmetries are shown in Fig. 2 ,

The problem of invariance to convolution can be expected much simplified when working in the Fourier domain. Convolution then becomes a simple multiplication. Given $f, h$ and $g$ are the original image, convolution kernel and the degraded image, resp.,

$$
g=f * h,
$$


and $F, H, G$ are their Fourier transforms, resp., then

$$
G=F H \text {. }
$$

If the convolution kernel $H(r, \varphi)$ (where $r, \varphi$ are polar coordinates) is having the $C_{n}$, resp. $D_{n}$, symmetry for $n=N$, then also its Fourier transform has the $C_{N}$, resp. $D_{N}$ symmetry.

When investigating rotational symmetries, it is useful to expand an image $\psi(r, \varphi)$ or its Fourier transform into the circular harmonics which are the basis of the 2-D rotation group representation [1]

$$
\psi(r, \varphi)=\sum_{m \in \mathbb{Z}} \psi_{m}(r) e^{i m \varphi}
$$

where the coefficients are

$$
\psi_{m}(r)=\frac{1}{2 \pi} \int_{0}^{2 \pi} \psi(r, \varphi) e^{-i m \varphi} d \varphi .
$$

\subsection{Relative Invariants}

We first introduce a relative invariant to convolution $I$, which applied on both the original and the degraded images $f, g$ stays the same upon a factor depending only on parameters of the convolution kernel

$$
I(g)=\Omega I(f) \quad \text { where } \quad \Omega=\Omega(h) .
$$

The simplest non-trivial relative invariant to convolution is Fourier transform of the image, as recalled in (4). If we find another invariant with $\Omega=H$, we can combine them to get an absolute invariant to convolution $(\Omega=1)$.

$\boldsymbol{C}_{\boldsymbol{n}}$ Symmetry. We can get inspired by investigating the $C_{n}$ symmetry. Fourier transforms of the original image, convolution kernel and the degraded image in (44) can be decomposed into circular harmonics basis as in (5). We will denote the coefficients in the expansions for $G, F$ and $H$ as $g_{i}(r), f_{i}(r)$ and $h_{i}(r)$, respectively. Given the convolution kernel $H(r, \varphi)$ has a $C_{n}$ symmetry for $n=N$. Then the coefficients $h_{m}$ are nonzero only for $m=k N, k \in \mathbb{Z}$ and we obtain the following relation.

$$
\sum_{s \in \mathbb{Z}} g_{s}(r) e^{-i s \varphi}=\sum_{m \in \mathbb{Z}} f_{m}(r) e^{-i m \varphi} \sum_{k \in \mathbb{Z}} h_{k N}(r) e^{-i k N \varphi}
$$

One particular coefficient $g_{s}(r)$ then equals

$$
g_{s}(r)=\sum_{k \in \mathbb{Z}} f_{s-k N}(r) h_{k N}(r) .
$$

It is obvious from (9), that a set of coefficients $\left\{f_{s-k N}\right\}$ for one particular $s$ is closed under convolution with the $C_{n}$ symmetry for $n=N$. Thus, it can be 
expected, that an invariant to $C_{N}$ symmetrical convolution should be based on harmonics from one of these subspaces. In other words, the linear space spanned by all the harmonics can be divided into $N$ independent subspaces, where each of them stays invariant under $C_{N}$ symmetrical convolution. We can also obtain them by applying projection operators to get irreducible subspaces for the $C_{N}$ symmetry group. For the $C_{N}$ group there are $N$ such operators

$$
\mathcal{P}^{m, N} F(r, \varphi)=\frac{1}{N} \sum_{k=0}^{N-1} F(r, \varphi-2 \pi k / N) e^{i m \frac{2 \pi k}{N}}
$$

for $m=0, \ldots, N-1$. The operator incrementally rotates $F$ by angle $2 \pi / N$ and a weighted average is calculated. Projection (10) applied on $F(r, \varphi)$ gives

$$
\mathcal{P}^{m, N} F(r, \varphi)=\sum_{k \in \mathbb{Z}} f_{k N+m}(r) e^{i(k N+m) \varphi} .
$$

Substituting (9) into (11) it is straightforward to get

$$
\mathcal{P}^{m, N} G(r, \varphi)=H \mathcal{P}^{m, N} F(r, \varphi) .
$$

Thus, we get another set of relative invariants to convolution with $C_{n}$ symmetry where $n=N$

$$
\mathcal{P}^{m, N} F(r, \varphi) \quad \text { where } \quad \Omega=H
$$

Projection Operators and $\boldsymbol{D}_{\boldsymbol{n}}$ Symmetry. The last conclusion can be also observed immediately from the projection operator. For every group, we can define projection operators projecting particular space into its irreducible subspaces [11]. In a simplified form the projection operator can be written

$$
\mathcal{P}^{\alpha}=\text { const }_{\alpha} \sum_{k} \chi^{\alpha}\left(G_{k}\right) T\left(G_{k}\right)
$$

where $G_{k}$ is an element of a particular symmetry group, $T\left(G_{k}\right)$ is a corresponding transformation operator and $\chi^{\alpha}\left(G_{k}\right)$ is simply a coefficient which can be found in a so called character table for a particular symmetry group [12].

Since convolution kernels discussed in this paper remain the same under all the operators (they are fully symmetric), they can be factored out. Thus projection on an arbitrary irreducible subspace applied in the Fourier transform of an image is a relative invariant with $\Omega=H$. The conclusions apply also particularly on both groups $C_{n}$ and $D_{n}$. This result is one of the main achievements of this paper. Although here we elaborate only on the two symmetry groups $C_{n}$ and $D_{n}$, the result is applicable to symmetries in an arbitrary dimension where we are able to formulate the symmetrizing projection operator. We can thus expect to derive in our future research e.g. invariants to a PSF in a 3D microscopy.

It has to be emphasized that the above result is valid for the dihedral symmetries with arbitrary axis orientation but this orientation must be known (to be able to define corresponding projection operators). Without loss of generality we further assume that the horizontal axis is one of the symmetry axes. 


\section{Expressing the Invariants in Terms of Complex Moments}

Working in the Fourier domain may not always be practical for pattern recognition tasks because of noisy high-frequency components. Hence, we find equivalent invariants in the spatial domain. We get inspired by the well known fact that Fourier transform can be expressed in terms of image moments, as we explain in the next Section. It finally leads to the invariants to convolution with a dihedral kernel expressed in terms of complex moments.

\subsection{Expansion of the Fourier Transform with Complex Moments}

In this section we derive relation between Fourier transform and the complex moments. Importance of this formula will become clear in the next sections.

Complex moments of an image $f(x, y)$ of order $r=p+q$

$$
c_{p q}^{(f)}=\int_{-\infty}^{\infty} \int_{-\infty}^{\infty}(x+i y)^{p}(x-i y)^{q} f(x, y) d x d y
$$

are used to investigate problems where a rotational symmetry plays its role. $i$ is the imaginary unit. (The polynomials which the image is projected onto are a basis of the representation of the rotational group $\mathrm{SO}(2)$ [1]]).

Fourier transform is in cartesian coordinates defined as

$$
F(u, v)=\iint f(x, y) e^{-2 \pi i(x u+y v)} d x d y .
$$

It is well known, that (16) can be expanded into power series, where the coefficients are moments - after expanding the exponential we get monomials which appear in the definition of image moments. Accordingly, we would like to rewrite (16) So that after expansion we get polynomials (powers of $x+i y$ and $x-i y$ ) appearing in the definition of complex moments (15). Therefore, we express both spatial and spectral coordinates $x, y$ and $u, v$ using substitutions

$$
\left(\begin{array}{l}
u \\
v
\end{array}\right)=\left(\begin{array}{cc}
1 & 1 \\
i & -i
\end{array}\right)\left(\begin{array}{l}
U \\
V
\end{array}\right) \quad \text { and } \quad\left(\begin{array}{l}
x \\
y
\end{array}\right)=\left(\begin{array}{cc}
1 / 2 & 1 / 2 \\
-i / 2 & i / 2
\end{array}\right)\left(\begin{array}{c}
X \\
Y
\end{array}\right)
$$

what results in

$$
F^{\prime}(U, V)=\iint f(x, y) e^{-2 \pi i(X U+Y V)} d x d y .
$$

Then (18) can be expanded into power series with complex moments

$$
F^{\prime}(U, V)=\sum_{k=0}^{\infty} \sum_{j=0}^{\infty} \frac{(-2 \pi i)^{k+j}}{k ! j !} c_{k, j} U^{k} V^{j}
$$


The derived formula gets clear interpretation, if we use polar spectral coordinates $r$ and $\varphi$ instead of the cartesian $u$ and $v$. The auxiliary variables can then be written as

$$
U=\frac{r}{2} e^{-i \varphi} \quad \text { and } \quad V=\frac{r}{2} e^{i \varphi}
$$

and the Fourier transform can be expressed as

$$
F(r, \varphi)=\sum_{k=0}^{\infty} \sum_{j=0}^{\infty} \frac{(-\pi i)^{k+j}}{k ! j !} r^{k+j} c_{k, j} e^{-i(k-j) \varphi} .
$$

\subsection{Derivation of Moment Invariants to Convolutions with Kernel Symmetries $C_{n}$ and $D_{n}$}

We recall that above we have found two relative invariants to symmetrical convolution. We can combine them to get an absolute invariant

$$
\frac{F(r, \varphi)}{\mathcal{P}^{S} F(r, \varphi)} \text {. }
$$

where $\mathcal{P}^{S}$ denotes the symmetrical projection for an arbitrary symmetrical group.

The absolute invariant (22) can be expanded into series containing either $U^{k} V^{j}$ from the expansion (19) or circular harmonics from the expansion (21). Thus (22) can be expanded into series

$$
\sum_{v=-\infty}^{\infty} \sum_{s=0}^{\infty} I_{v, s} r^{s} e^{i v \varphi} \quad \text { or } \quad \sum_{k=0}^{\infty} \sum_{j=0}^{\infty} I_{k, j} U^{k} V^{j}
$$

where the coefficients $I_{v, s}$ or $I_{k, j}$ must be also absolute invariants. The symmetrical projection can be easily expressed using symmetry considerations. E.g. for the $C_{n}$ symmetry and $n=N$ the symmetrical projection equals

$$
\sum_{d=-\infty}^{\infty} \psi_{N d}(r) e^{i N d \varphi}
$$

By comparing the same monomials and powers of harmonics, we derive the following invariants $C_{p, q}^{N}$, resp. $D_{p, q}^{N}$ to the $C_{n}$, resp. $D_{n}$ symmetrical convolutions, where $n=N$ is the symmetry order. (They differ from the invariant coefficients in (23) only by constants.)

$$
\begin{gathered}
C_{p q}^{N}=\frac{c_{p q}}{c_{00}}-\sum_{\substack{j=0 \\
0<j+k}}^{p} \sum_{\substack{k=0 \\
(j-k) / N \in \mathbb{Z}}}^{q}\left(\begin{array}{l}
p \\
j
\end{array}\right)\left(\begin{array}{l}
q \\
k
\end{array}\right) \frac{c_{j k}}{c_{00}} C_{p-j, q-k}^{N} \\
D_{p q}^{N}=\frac{c_{p q}}{c_{00}}-\sum_{\substack{j=0 \\
0<j+k}}^{p} \sum_{\substack{k=0 \\
(j-k) / N \in \mathbb{Z}}}^{q}\left(\begin{array}{l}
p \\
j
\end{array}\right)\left(\begin{array}{l}
q \\
k
\end{array}\right) \frac{\Re c_{j k}}{c_{00}} D_{p-j, q-k}^{N}
\end{gathered}
$$




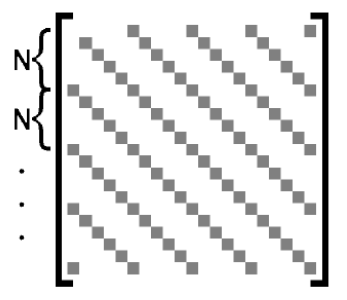

Fig. 3. Strip structure of the matrix of $C^{N}$. Grey elements correspond to trivial invariants.

where $\Re$ denotes real part of a complex number. Note that the summation goes only over such indices that $(j-k) / N$ is integer. This is because $c_{j k}^{(h)}=0$ for non-integer $(j-k) / N$ (see [9] for the proof).

We recall that (26) is derived for particular convolution kernel orientation, where one of the axial symmetry axes coincides with the horizontal axis. If the kernel is rotated by an arbitrary (but known) angle $\theta$, (26) changes to

$$
D_{p q}^{N}=\frac{c_{p q}}{c_{00}}-\sum_{\substack{j=0 \\
0<j+k}}^{p} \sum_{\substack{k=0 \\
(j-k) / N \in \mathbb{Z}}}^{q}\left(\begin{array}{l}
p \\
j
\end{array}\right)\left(\begin{array}{l}
q \\
k
\end{array}\right) \frac{c_{j k}+c_{k j} e^{i 2 \theta(j-k)}}{2 c_{00}} D_{p-j, q-k}^{N}
$$

Invariants $C_{p q}^{N}$ are trivial (they equal zero) for such $N, p, q$ where $(p-q) / N$ is integer. Hence, the matrix of $C^{N}$ has a strip structure (see Fig. 33) with zeros on the main and certain minor diagonals. The matrix of $D^{N}$ has the same structure, with zeros on the main diagonal, but with non-zero invariants on the minor diagonals. The existence of these valid invariants is the major advantage of $D^{N}$ set over the $C^{N}$ set.

\section{Experiments}

In this section we demonstrate invariance and discriminability of the newly derived invariants. It contains experiments with both simulated and real degradations of real photographs. However, focus is on experiments with real data to show the performance of the new invariants.

All the photographs used in the experiments were taken with the Nikon D5100 camera and with the AF-S DX NIKKOR 18-55mm f/3.5-5.6G VR objective. We experiment only with the green color channel for simplicity. We thus avoid difficulties related to possible chromatic aberration effects. Direct conversion to gray scale might violate our simple image degradation model.

\subsection{Simulated Degradation with Known Ground Truth}

The objective of the first experiment is to experimentally prove invariance of the new features on known ground truth data. We take patches (with sufficient 
variability) from ten real photographs, we convolve them on a computer with a symmetrical kernel of several sizes and several different orders of symmetry and we evaluate the invariants on both the original and the blurred patches. We repeat every test on 100 different randomly picked patches with diameters $51 \mathrm{px}$. Moments and invariants of orders 5 were used. Invariants $C^{N}$ and $D^{N}$ calculated on the original and degraded patches were compared and their mean relative error (MRE) was calculated. Relative error between numbers $x$ and $y$ is calculated as $(|x-y|) /(|x|+|y|+\epsilon)$ where $\epsilon$ is the machine precision.

Symmetry orders $N=2$ and $N=4$ were excluded from the evaluation since such symmetries coincide with the symmetry of the natural image pixel grid and we get very low errors comparable with machine precision. Most of the MRE's were lower than $0.01,99 \%$ are lower than 0.02 . This indicates good invariance of the features. Typical MRE calculated on two different images is higher than 0.1, what is on the other hand an indication of good discriminability of the features.

\subsection{Real Template Matching}

The objective of this experiment is to show that the newly proposed features are performing better than current available invariants to convolution. On a pair of real photographs we perform patch matching and we use the results to register them. A photograph of a painting was taken first sharp and then it was taken intentionally defocused. Small aperture was used such that the aperture contour is polygonal. Its symmetry is $D_{7}$ based on the number of aperture blades. We thus expect that $D^{7}$ invariants should perform best on these photographs. We took special care to prevent geometrical distortion of the photographs. Therefore, we also compensated for changes in scale when re-focusing the camera. Our goal was not to challenge rotation or scale invariance. We wanted to focus mainly on evaluation of added value of the $D^{7}$ invariants compared to other invariants. The photographs are relatively noisy due to low-light shooting conditions.

Both the sharp image and the de-focused image are shown in Fig. 4 together with ten manually selected feature points and with the templates around them. We took every template and looked by exhaustive search in the whole blurred image for the most similar (in terms of MRE between the invariants) counterpart. We tested various invariants in this task, see Table 1- $-C^{2}, C^{3}, C^{7}$, and $C^{\infty}$ invariants to kernels with $N$-fold rotation symmetry and $D^{2}, D^{3}, D^{7}$ invariants to kernels with dihedral symmetry. For comparison we also calculated ordinary moments $M$, which are not invariant to any blur. All features are evaluated using different moment orders, see the top row of Table 1.

Once the best counterpart was found in the degraded image, its position was recorded. Since the ground-truth match is not known, the evaluation was done by means of image registration. The original points and the corresponding matching points were used to estimate the affine transform mapping between them minimizing least squares error. The original points were transformed using the affine transform fit and the standard deviation was calculated between positions of the transformed original points and the detected matching points. All of them are shown in the Table 1 . 

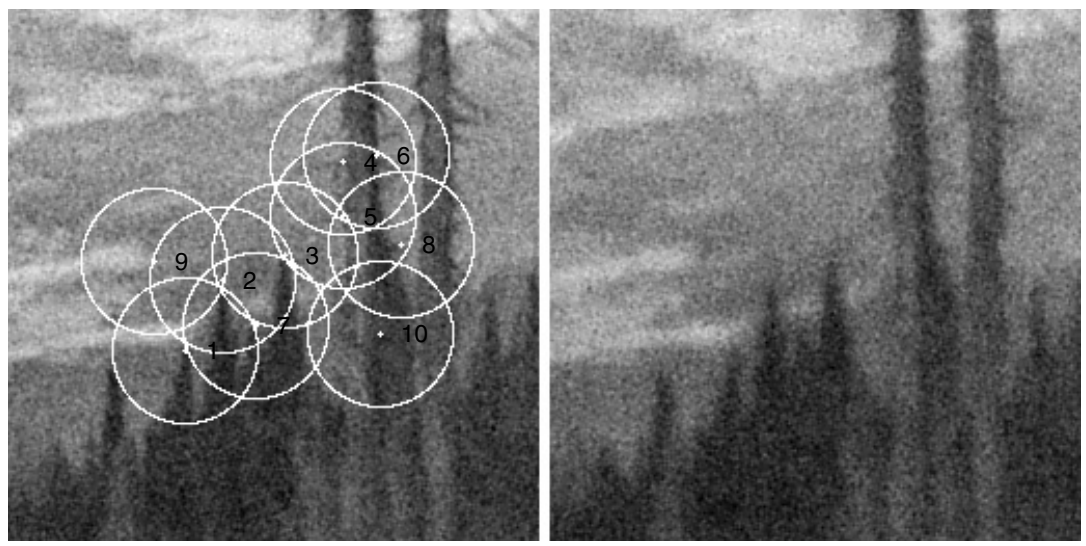

Fig. 4. Left: Original sharp photograph (segment) with manually chosen control points with depicted corresponding patches. Right: De-focused photograph (segment).

Table 1. Standard deviations (see text) as a performance measure of listed features (left column)

\begin{tabular}{|l|c|c|c|c|c|}
\hline kernel / order & 3 & 4 & 5 & 6 & 7 \\
\hline$C_{2}$ & 38.6 & 14.8 & 18.7 & 4.19 & 4.19 \\
$C_{3}$ & 2.77 & 2.80 & 2.32 & 1.88 & 1.88 \\
$C_{7}$ & 25.8 & 3.83 & 3.30 & 1.98 & 1.98 \\
$D_{2}$ & 14.1 & 2.40 & 2.39 & 20.9 & 20.9 \\
$D_{3}$ & 5.22 & 4.76 & 2.89 & 2.03 & 2.03 \\
$D_{7}$ & 5.22 & 4.05 & 2.27 & 1.56 & 1.56 \\
$C_{\infty}$ & 36.9 & 32.0 & 24.3 & 13.9 & 3.30 \\
$M$ & 36.8 & 25.2 & 9.56 & 35.5 & 4.65 \\
\hline
\end{tabular}

We can see that the invariants $D^{7}$ perform best, as expected, if we use sufficient moment order. Although $C^{7}$ features also work well and they are also invariant to the experimental convolution kernel, we can see that higher number of available features $D^{7}$ further improves the result. Pure ordinary moments perform poorly, which indicates that the blur size is large enough and we cannot successfully match the patches without using the invariants to convolution.

\section{Conclusions}

We have derived general formulas for invariants to convolution with symmetrical kernels in an arbitrary dimension. In 2D and for specific dihedral symmetry $D_{n}$ we have expressed the invariants in terms of image moments, making thus the theory easily applicable in practice.

The main advantage of $D_{n}$ invariants over the earlier $C_{n}$ invariants is that there exist more of them and hence they provide higher discrimination power. On the other hand, a serious limitation is that the orientation of dihedral axes 
must be known while the usage of the $C_{n}$ invariants does not require it. This limitation can be possibly overcome by estimating the kernel orientation directly from the degraded image, which will be a topic of our future research.

Acknowledgments. The authors express their gratitude to the Czech Science Foundation for financial support of this work under the grant No. P103/11/1552.

\section{References}

1. Flusser, J., Suk, T.: Degraded image analysis: An invariant approach. IEEE Trans. on Pattern Analysis and Machine Intelligence 20(6), 590-603 (1998)

2. Bentoutou, Y., Taleb, N., Kpalma, K., Ronsin, J.: An automatic image registration for applications in remote sensing. IEEE Trans. on Geoscience and Remote Sensing 43(9), 2127-2137 (2005)

3. Liu, Z., An, J., Li, L.: A two-stage registration angorithm for oil spill aerial image by invariants-based similarity and improved ICP. International Journal of Remote Sensing 32(13), 3649-3664 (2011)

4. Hu, S.X., Xiong, Y.M., Liao, M.Z.W., Chen, W.F.: Accurate point matching based on combined moment invariants and their new statistical metric. In: Proc. of the 2007 Int. Conf. on Wavelet Analysis and Pattern Recognition, ICWAPR 2007, Beijing, China, pp. 376-381. IEEE (2007)

5. Bentoutou, Y., Taleb, N., Chikr El Mezouar, M., Taleb, M., Jetto, L.: An invariant approach for image registration in digital subtraction angiography. Pattern Recognition 35(12), 2853-2865 (2002)

6. Tang, S., Wang, Y., Wei Chen, Y.: Blur invariant phase correlation in X-ray digital subtraction angiography. In: Proc. of the Int. Conf. on Complex Medical Engineering, CME 2007, pp. 1715-1719. IEEE (May 2007)

7. Bentoutou, Y., Taleb, N.: A 3-D space-time motion detection for an invariant image registration approach in digital subtraction angiography. Computer Vision and Image Understanding 97, 30-50 (2005)

8. Flusser, J., Zitová, B.: Invariants to convolution with circularly symmetric PSF. In: Proc. of the 17th Int. Conf. on Pattern Recognition, ICPR 2004, pp. 11-14. IEEE Computer Society (2004)

9. Flusser, J., Suk, T., Zitová, B.: Moments and Moment Invariants in Pattern Recognition. Wiley, Chichester (2009)

10. Flusser, J., Suk, T., Boldyš, J., Zitová, B.: Invariants to image blurring. IEEE Transactions on Pattern Analysis and Machine Intelligence (revised)

11. Elliot, J.P., Dawber, P.G.: Symmetry in Physics, vol. 1. Oxford University Press, Oxford (1985)

12. Bradley, C., Cracknell, A.: The mathematical theory of symmetry in solids: representation theory for point groups and space groups. Clarendon Press (1972) 